\title{
Detection of somatic mutations of the PIG-A gene in Brazilian patients with paroxysmal nocturnal hemoglobinuria
}

R. Franco de Carvalho,

V.R. Arruda, S.T.O. Saad

and F.F. Costa
Centro de Hematologia e Hemoterapia - Hemocentro,

Universidade Estadual de Campinas, Campinas, SP, Brasil
Correspondence

F.F. Costa

Hemocentro, Unicamp

Caixa Postal 6198

13081-970 Campinas, SP

Brasil

E-mail: ferreira@ unicamp.br

Research supported by CAPES.

Publication supported by FAPESP.

Received January 10, 2001 Accepted April 10, 2001

\section{Abstract}

Paroxysmal nocturnal hemoglobinuria $(\mathrm{PNH})$ is an acquired clonal syndrome characterized by intravascular hemolysis mediated by complement, thrombotic events and alterations in hematopoiesis. Basically, the molecular events which underlie the complexity of the syndrome consist of the absence of the glycosylphosphatidylinositol (GPI) anchor as a consequence of somatic mutations in the $P I G-A$ gene, located on the $\mathrm{X}$ chromosome. The GPI group is responsible for the attachment of many proteins to the cytoplasmic membrane. Two of them, CD55 and CD59, have a major role in the inhibition of the action of complement on the cellular membrane of blood cells. The absence of GPI biosynthesis can lead to PNH. Since mutations in the $P I G-A$ gene are always present in patients with $\mathrm{PNH}$, the aim of this study was to characterize the mutations in the $P I G-A$ gene in Brazilian patients. The analysis of the $P I G-A$ gene was performed using DNA samples derived from bone marrow and peripheral blood. Conformationsensitive gel electrophoresis was used for screening the mutation and sequencing methods were used to identify the mutations. Molecular analysis permitted the identification of three point mutations in three patients: one $\mathrm{G} \rightarrow \mathrm{A}$ transition in the 5' portion of the second intron, one $\mathrm{T} \rightarrow \mathrm{A}$ substitution in the second base of codon 430 (Leu430 $\rightarrow$ stop), and one deletion $\Delta \mathrm{A}$ in the third base of codon 63 . This study represents the first description of mutations in the $P I G-A$ gene in a Brazilian population.

\section{Introduction}

Paroxysmal nocturnal hemoglobinuria (PNH) is an acquired hematological syndrome characterized by chronic intravascular hemolysis mediated by complement, thrombotic events and alterations in hematopoiesis (1-3). The molecular alterations which underlie the complexity of the syndrome are relatively simple (4-7). Basically, they consist of the absence of the glycosylphosphati-

\section{Key words}

- Paroxysmal nocturnal hemoglobinuria

- Intravascular hemolysis

- Complement PIG-A

- Mutation dylinositol (GPI) group, responsible for the attachment of several proteins in the cytoplasmic membrane (8), as a consequence of somatic mutations in the $P I G-A$ gene located on the short arm of the X chromosome $(9,10)$.

The GPI group attaches about 20 proteins to the blood cell's membrane (6). At least two of them, CD55 (decay accelerating factor) and CD59 (membrane inhibitor of reactive lysis), are involved in the inhibition of the action of complement on the blood cell 
membrane. Thus, the absence of GPI biosynthesis can lead to PNH.

Since mutations in the $P I G-A$ gene are always present in patients with PNH (1116), and to the best of our knowledge there is no previous report of these mutations in Brazil, the aim of the present study was to characterize somatic mutations in the $P I G-A$ gene in Brazilian patients with PNH.

\section{Material and Methods}

Three PNH patients ( 1 female aged 25 years and 2 males aged 17 and 65 years) were investigated. The diagnosis of $\mathrm{PNH}$ was based on clinical and laboratory features (1). This study was approved by the Ethics Committee (Hemocentro, UNICAMP) and the patients gave informed written consent. The quantification of GPI-linked surface proteins on red blood cells and granulocytes was performed by flow cytometry

Table 1. Mutations detected in patients with paroxysmal nocturnal hemoglobinuria (PNH).

\begin{tabular}{lccccc}
\hline Patients & $\begin{array}{c}\text { Age } \\
\text { (years) }\end{array}$ & Sex & $\begin{array}{c}\text { Altered fragment* } \\
\text { (CSGE scan) }\end{array}$ & $\begin{array}{c}\text { Mutation } \\
\text { (codon) }\end{array}$ & Type \\
\hline PNH1 & 25 & Female & Fragment 2B & agg $\rightarrow$ aga & Splicing defect \\
PNH2 & 17 & Male & Fragment 6A & 430TTG $\rightarrow$ TAG & Nonsense \\
PNH3 & 65 & Male & Fragment 2A & 63GAG $\rightarrow$ G_G & Frameshift
\end{tabular}

*The amplification fragments were those described by lida et al. (see Ref. 10). CSGE, conformation-sensitive gel electrophoresis.

Figure 1. Nucleotide sequence of fragment $2 B$ with a $G \rightarrow A$ substitution in the $5^{\prime}$ portion (arrow) of the second intron found in patient $\mathrm{PNH}$. From left to right: control and the abnormal sequence derived from the patient's bone marrow sample. $\mathrm{PNH}$, paroxysmal nocturnal hemoglobinuria.

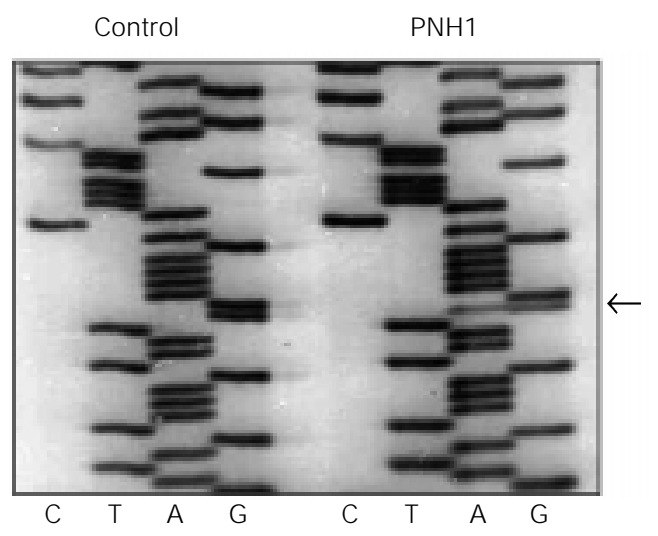

(FACScan; Becton-Dickson, Mountain View, CA, USA) using monoclonal antibodies towards GPI-linked surface antigens (CD59 and CD55). Peripheral venous blood and bone marrow samples were collected with EDTA as anticoagulant after obtaining informed patient consent. Genomic DNA derived from these samples was extracted by the Trisol method (Gibco-BRL, Rockville, MD, USA), which involves a monophasic reagent with a phenol and guanidine isothiocyanate solution. Contaminating erythrocytes in the preparations were removed by hypotonic lysis.

The promoter and coding regions (including exon/intron boundaries) of the $P I G$ $A$ gene were amplified from genomic DNA using six pairs of primers for 35 cycles (10). The PCR products were screened for mutations by conformation-sensitive gel electrophoresis (CSGE), a variation of the method of heteroduplex analysis (17). The mutations were identified by direct sequencing with the Sequenase kit version 2.0. When necessary, the PCR products were cloned in the pUC18 plasmid and sequenced.

\section{Results}

CSGE analysis and sequencing permitted the detection of the mutation in each of the three patients. The results are given in Table 1.

The first mutation identified in fragment $2 \mathrm{~B}$ (patient $\mathrm{PNH} 1$ ) was a $\mathrm{G} \rightarrow \mathrm{A}$ transition in the second intron 5' portion (Figure 1, indicated by the arrow). The second mutation detected in fragment $6 \mathrm{~A}$ (patient $\mathrm{PNH} 2$ ) was a $\mathrm{T} \rightarrow \mathrm{A}$ transversion in the second base of codon 430 (Figure 2). The identification of the third mutation, a deletion of one nucleotide $\mathrm{A}$ in the third base position of codon 63 (fragment 2A; patient PNH3), was only possible after subcloning the PCR product in a bacterial vector, which allowed the separation of the normal and mutant sequences (Figure 3). 


\section{Discussion}

The mutations detected in patients $\mathrm{PNH} 1$ and $\mathrm{PNH} 2$ have not been described in the literature. The first one, the $\mathrm{G} \rightarrow \mathrm{A}$ substitution in the conserved region at the 5 ' extremity of the second intron, is possibly disturbing the normal splicing mechanism in the mutant gene. The latter is a $\mathrm{T} \rightarrow \mathrm{A}$ substitution in the second base of codon Leu430 in exon 6 , leading to the formation of one precocious stop codon in this position (nonsense mutation). Due to this, at least 54 amino acids are not expressed. This region corresponds to two distinct protein domains, and by homology to other proteins, the region between amino acids 422 and 442 is described as a transmembrane domain. The region between amino acids 443 and 484 probably corresponds to the portion of the protein located in the lumen of the endoplasmic reticulum (http://www.expasy.ch). In this case, the resulting peptide may not be completely dysfunctional since the enzymatic domains seem to be located in the second exon (18). The third mutation (patient PNH3) has already been described (14). The loss of one nucleotide $\mathrm{A}$ in the third base of the Arg63 codon is responsible for the production of one truncated peptide with only 67 amino acids.

Somatic mutations in the $P I G-A$ gene are heterogeneous, without defined etiological characteristics. More than 148 mutations have been described (16), spanning the entire locus. These are mainly frameshifts involving one or two nucleotides $(5,14)$. Point mutations occurred in approximately $37 \%$ of the PNH cases studied, with a majority of missense mutations (5). However, comparative observation indicates discrepancies in the frequencies of the emergency of mutation types (frameshifts versus substitutions) in

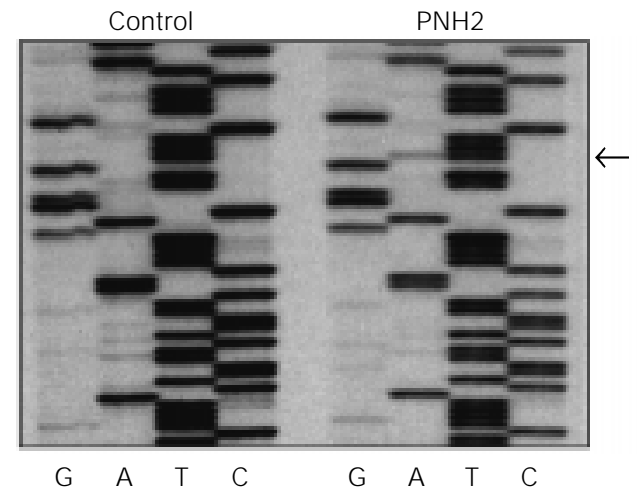

Figure 2. Nucleotide sequence of fragment $6 \mathrm{~A}$ with a $\mathrm{T} \rightarrow \mathrm{A}$ substitution in the second base of codon Leu430 (arrow) in patient $\mathrm{PNH} 2$. From left to right: control and the sample derived from bone marrow showing the abnormal sequence. $\mathrm{PNH}$, paroxysmal nocturnal hemoglobinuria.

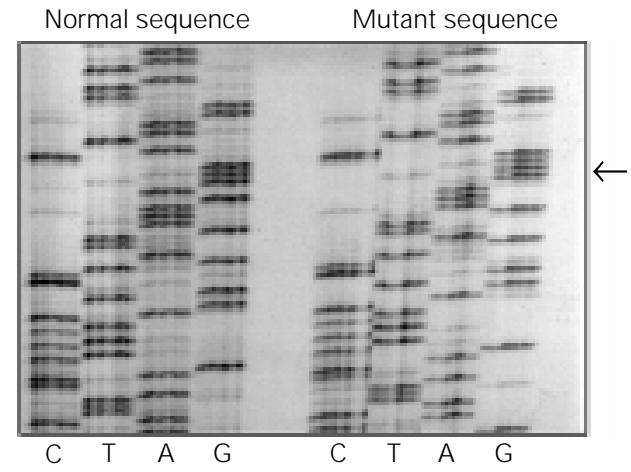

Figure 3. From left to right: normal and variant sequences derived from fragment $2 \mathrm{~A}$ of $\mathrm{pa}$ tient PNH3. The deletion of one nucleotide $A$ in the third base of the Arg63 codon (arrow) is responsible for the production of one truncated peptide with only 67 amino acids (14). different populations analyzed (12-14). Differences in the mutational spectra of the $P I G-A$ gene may be related to the specific genetic background of these populations and/ or to previous exposure to genotoxic agents $(19,20)$.

This study represents the first description of somatic mutations in the $P I G-A$ gene in a Brazilian population. In this respect, analysis of somatic mutations in the $P I G-A$ gene in Brazil could represent a new source of comparative mutation data. The results obtained suggest the emergence of two novel point mutations in Brazilian patients with $\mathrm{PNH}$. However, in view of the small number of patients, further studies are needed to obtain a comprehensive mutation profile of the $P I G$ $A$ gene in Brazil. 


\section{References}

1. Rosse WF (1995). Paroxysmal nocturnal hemoglobinuria. In: Handin RI, Stossel TP $\&$ Lux SE (Editors), Blood: Principles and Practice of Hematology. J.B. Lippincott, Philadelphia.

2. Hillmen $P$, Lewis $S M$, Bessler M, Luzzatto L \& Dacie JV (1995). Natural history of paroxysmal nocturnal hemoglobinuria. New England J ournal of Medicine, 333: 1253-1258.

3. Griscelli-Bennaceur A, Gluckman E, Scrobohaci ML, J onveaux T, Bazarbachi A, Carossella ED, Sigaux $F \&$ Socié $G$ (1995). Aplastic anemia and paroxysmal nocturnal hemoglobinuria: search for a pathogenetic link. Blood, 85: 1354-1363.

4. Luzzatto L \& Bessler M (1996). The dual pathogenesis of paroxysmal nocturnal hemoglobinuria. Current Opinion in Hematology, 3: 101-110.

5. Rosse WF \& Ware RE (1995). The molecular basis of paroxysmal nocturnal hemoglobinuria. Blood, 86: 3277-3286.

6. Rosse WF (1997). Paroxysmal nocturnal hemoglobinuria as a molecular disease. Medicine, 76: 63-93.

7. Rosti $\vee(2000)$. The molecular basis of paroxysmal nocturnal hemoglobinuria. Haematologica, 85: 82-87.

8. Cross GAM (1990). Glycolipid anchoring of plasma membrane proteins. Annual Review of Cell Biology, 6: 1-39.

9. Miyata T, Kawagoe K, lida Y, Endo Y, Fujita T, Takahashi M \& Kitani T (1993). Deficiency of GPI anchor caused by a somatic mutation of the PIG-A gene in paroxysmal nocturnal hemoglobinuria. Cell, 73: 703711.

10. lida $Y$, Takeda J, Miyata $T$, Inoue N, NishimuraJ , Kitani T, Maeda K \& Kinoshita T (1994). Characterization of genomic PIGA gene: A gene for glycosylphosphatidylinositol-anchor biosynthesis and paroxysmal nocturnal hemoglobinuria. Blood, 83 : 3126-3131.

11. Ware RE, Rosse WF \& Howard TA (1994). Mutations within the PIG-a gene in patients with paroxysmal nocturnal hemoglobinuria. Blood, 83: 2418-2422.

12. Yamata N, Miyata T, Maeda K, Kitani T, Takeda J \& Kinoshita T (1995). Somatic mutations of PIG-A gene found in J apanese patients with paroxysmal nocturnal hemoglobinuria. Blood, 85: 885-892.

13. Pramoonjago $\mathrm{P}$, Wanachiwanawin $\mathrm{W}$, Chinprasertzak S, Pattanapanayasat K, Takeda J \& Kinoshita T (1995). Somatic mutations of PIG-A in Thai patients with paroxysmal nocturnal hemoglobinuria. Blood, 86: 1736-1739.

14. Nafa $K$, Mason PJ, Hillmen $P$, Luzzatto $L$ $\&$ Bessler M (1995). Mutations in PIG-a gene causing paroxysmal nocturnal hemoglobinuria are mainly of the frameshift type. Blood, 86: 4650-4655.

15. Nagarajan S, Brodsky RA, Young NS \& Medof ME (1995). Genetic defects underlying paroxysmal nocturnal hemoglobinuria that arises out of aplastic anemia. Blood, 86: 4656-4661.
16. Nafa $\mathrm{K}$, Bessler M, Castro-Malaspina $\mathrm{H}$ J hanwar S \& Luzzatto L (1998). The spectrum of somatic mutations in PIG-A gene in paroxysmal nocturnal hemoglobinuria includes large deletions and small duplications. Blood Cells, Molecules, and Diseases, 24: 370-384.

17. Ganguly A, Rock MJ \& Prockpop DJ (1993). Conformation-sensitive gel electrophoresis for rapid detection of singlebase differences in double-stranded PCR products and DNA fragments: evidence for solvent-induced bends in DNA heteroduplexes. Proceedings of the National Academy of Sciences, USA, 20: 1032510329.

18. Norris ER, Howard TA, Marcus SJ \& Ware RE (1997). Structural and functional analysis of the PIG-A protein that is mutated in paroxysmal nocturnal hemoglobinuria. Blood Cells, Molecules, and Diseases, 23: 350-360.

19. Purow DA, HowardTA, Marcus SJ , Rosse WF \& Ware RE (1999). Genetic instability and the etiology of somatic mutations in paroxysmal nocturnal hemoglobinuria. Blood Cells, Molecules, and Diseases, 25: 79-89.

20. Glickman BW, Kotturi G, de Boer J \& Kusser W (1995). Molecular mechanisms of mutagenesis and the mutational spectra. In: Venitt S \& Phillips DH (Editors), Enviromental Mutagenesis. BIOS Scientific Publishers Limited, Oxford, UK. 